\title{
The Electric Field of ATP-Synthase
}

\section{Jean-Nicolas Vigneau', Peyman Fahimi ${ }^{1,2}$, Maximilian Ebert ${ }^{3}$, Youji Cheng ${ }^{2,4}$, Chérif F. Matta ${ }^{2,4}$}

${ }^{1}$ Département de chimie, Université Laval, Québec, QC, Canada G1V0A6, ${ }^{2}$ Department of Chemistry and Physics, Mount Saint Vincent University, Halifax, NS, Canada B3M2J6, ${ }^{3}$ Chemical Computing Group (CCG), Sherbrooke Street West, Montreal, QC, Canada H3A2R7, ${ }^{4}$ Department of Chemistry, Saint Mary's University, Halifax, NS, Canada B3H3C3.

\section{cherif.matta@msvu.ca}

This talk will underscore the importance of developing fast Quantum Crystallographic (QCr) [1] approaches to accurately calculate the electric fields and their associated electrostatic potentials of large molecules such as proteins. Crystallographic structures of ATP synthase from five species have been used to calculate (approximately) and compare their intrinsic electrostatic potentials and fields [2-4]. Striking consistent patterns (and differences) in the topographies of these scalar and vector fields are uncovered across the five studied ATP synthases [2]. The role of these fields in the biological function of ATP synthase will be discussed within the context of Mitchell's chemisomotic theory. Our calculations suggest that, due to the intrinsic field of ATP synthase itself, the standard equation of chemiosmotic must be augmented by including a term that accounts for the contribution to $\Delta G$ of the difference in ATP synthase's electrostatic potential, $\Delta \psi_{\text {ATPase }}$, between the points of entry and exit of the protons in the mitochondrion. With this inclusion, our proposed amended equation for the chemisomotic $\Delta G$, in standard notation, becomes [2-4]:

$$
\Delta G=\Delta G_{\text {chem. }}+\Delta G_{\text {elec. }} \underbrace{+\Delta G_{\text {ATPase }}}_{\text {NEW TERM }}=2.3 n R T \Delta \mathrm{pH}+n \mathscr{F} Z \Delta \psi+\underbrace{n \mathscr{F} Z \Delta \psi_{\text {ATPase }}}_{\text {NEW TERM }}
$$

Our results can be summed-up into assigning two separate but complementary roles to ATP synthase [2]:

(1) Its putative role, and that is the catalysis (i.e. lowering the $\Delta G^{\ddagger}$ ) of the reaction: $\mathrm{ADP}+\mathrm{P}_{\mathrm{i}} \rightleftharpoons \mathrm{ATP}+\mathrm{H}_{2} \mathrm{O}$.

(2) A novel role, that is, of altering the $\Delta G$ of the reaction of translocation of protons from the inter-membrane gap in the mitochondrion to the mitochondrial matrix, i.e. the reactions: $\mathrm{H}_{\text {inter-membrane space }} \rightleftharpoons \mathrm{H}_{\text {matrix }}^{+}$.

Said differently, due to the enzyme's very structure and due to the chemiosmotic origin of the free energy it harnesses, ATP synthase functions over an above its role as an enzyme and is more than strictly a biological catalyst.

The crucial role played by the enzyme's own electric properties calls for their accurate and fast determinations especially with the advent of QCr [1]. An example of such approaches is the Kernel Energy Method [5-6] fragmentation whereby, given a molecular geometry, one can perform quantum calculations on fragments and obtain an approximate total electrostatic potential of the full molecules. Since this is a part of a larger project, time permitting, the talk may touch upon some of the hot current open questions such as the one we term "Mitochondrion Paradox" [7-10].

[1] Genoni, A. ; Bucinskż, L.; Claiser, N.; Contreras-Garcia, J.; Dittrich, B.; Dominiak, P. M.; Espinosa, E.; Gatti, C.; Giannozzi, P.; Gillet, J.M.; Jayatilaka, D.; Macchi, P.; Madsen, A. U.; Massa, L.; Matta, C. F.; Merz Jr., K. M.; Nakashima, P.; Ott, H.; Ryde, U.; Scherer, W.; Schwarz, K.; Sierka, M.; Grabowsky, S. (2018) Chem. Eur. J. 24, 10881-10905.

[2] Vigneau, J. N.; Fahimi, P.; Ebert, M.; Cheng, Y.; Tannahill, C.; Muir, P.; Nguyen-Dang, T.-T.; Matta, C. F. (2021) Submitted, in review.

[3] Cheng, Y. (2019). A Computational Investigation of the Intrinsic Electric Field of ATP Synthase (M.Sc. Thesis); Saint Mary's University: Halifax, Canada.

[4] Matta, C. F. (2016). Dipolar field of ATP Synthase: Unpublished results presented in a number of seminars.

[5] Huang L.; Massa, L.; Karle, J. (2010). Chapter 1 in: Quantum Biochemistry: Electronic Structure and Biological Activity, Vol. I; Matta, C. F. (Ed.), Wiley-VCH: Weinheim, 2010.

[6] Polkosnik, W.; Matta, C. F.; Huang, L.; Massa, L. (2019). Int. J. Quantum Chem. 119, e26095.

[7] Fahimi, P. ; Matta, C. F. (2021). Phys. Biol. 18 , in press (DOI: 10.1088/1478-3975/abf7d9).

[8] Fahimi, P. ; Matta, C. F. (2021) Submitted, in review.

[9] Nasr, M. A.; Dovbeshko, G. I.; Bearne, S. L.; El-Badri, N.; Matta, C. F. (2019). BioEssays 41, 1900055.

[10] Matta, C. F.; Massa, L. (2017). J. Phys. Chem. A 121, 9131-9135.

Keywords: ATP synthase; chemiosmotic theory; mitochondrial biophysics; electric field; electrostatic potential 